\title{
BMJ Open The productivity development of total hip arthroplasty in Sweden: a multiple registry-based longitudinal study using the Malmquist Productivity Index
}

\author{
Fanny Goude, ${ }^{\oplus 1,2}$ Göran Garellick, ${ }^{3}$ Sverre A C Kittelsen, ${ }^{4}$ Szilard Nemes, ${ }^{5}$ \\ Clas Rehnberg ${ }^{1,2}$
}

To cite: Goude F, Garellick G, Kittelsen SAC, et al. The productivity development of total hip arthroplasty in Sweden: a multiple registry-based longitudinal study using the Malmquist Productivity Index. BMJ Open 2019;9:e028722. doi:10.1136/ bmjopen-2018-028722

- Prepublication history and additional material for this paper are available online. To view these files, please visit the journal online (http://dx.doi org/10.1136/bmjopen-2018028722).

Received 20 December 2018 Revised 17 July 2019 Accepted 19 July 2019

\section{Check for updates}

(c) Author(s) (or their employer(s)) 2019. Re-use permitted under CC BY-NC. No commercial re-use. See rights and permissions. Published by BMJ.

${ }^{1}$ Department of Learning, Informatics, Management and Ethics, Karolinska Institutet, Stockholm, Sweden

${ }^{2}$ Centre for Health Economics, Informatics and Health Services Research, Stockholm Health Care Services, Stockholm,

Sweden

${ }^{3}$ Swedish Hip Arthroplasty Register, Gothenburg, Sweden ${ }^{4}$ Frisch Centre, Oslo, Norway

${ }^{5}$ Department of Orthopaedics, Sahlgrenska Academy, University of Gothenburg, Gothenburg, Sweden

Correspondence to

Fanny Goude;

fanny.goude@ki.se

\section{ABSTRACT}

Objective The increasing demand for total hip arthroplasty (THA) combined with limited resources in healthcare puts pressure on decision-makers in orthopaedics to provide the procedure at minimum costs and with good outcomes while maintaining or increasing access. The objective of this study was to analyse the development in productivity between 2005 and 2012 in the provision of THA.

Design The study was a multiple registry-based longitudinal study.

Setting and participants The study was conducted among 65 orthopaedic departments providing THA in Sweden from 2005 to 2012.

Outcome measures The development in productivity was measured by Malmquist Productivity Index by relating department level total costs of THA to the number of noncemented, hybrid and cemented THAs. We also break down the productivity change into changes in efficiency and technology.

Results Productivity increased significantly in three periods (between $1.6 \%$ and $27.0 \%$ ) and declined significantly in four periods (between $0.8 \%$ and $12.1 \%$ ). Technology improved significantly in three periods (between $3.2 \%$ and $16.9 \%$ ) and deteriorated significantly in two periods (between $10.2 \%$ and $12.6 \%$ ). Significant progress in efficiency was achieved in two periods (ranging from $2.6 \%$ to $8.7 \%$ ), whereas a significant regress was attained in one period (3.9\%). For the time span as a whole, an average increase in productivity of $1.4 \%$ per year was found, where changes in efficiency contributed more to the improvement (1.1\%) than did technical change $(0.2 \%)$.

Conclusions We found a slight improvement of productivity over time in the provision of THA, which was mainly driven by changes in efficiency. Further research is, however, needed where differences in quality of care and patient case mix between departments are taken into account.

\section{INTRODUCTION}

Total hip arthroplasty (THA) is a common surgical procedure worldwide, with increasing incidence. In Sweden, between 1980 and 2010, the annual number of THAs more

\section{Strengths and limitations of this study}

- The approach we took in analysing the productivity development has to our knowledge never been used previously in the field of total hip arthroplasty (THA)

- We made use of unique patient-level data from several national patient registers covering all orthopaedic departments in Sweden over 8 years.

- We provide insight into the development of productivity, efficiency and technology in the provision of THA, which can assist managers and policymakers in undertaking measures for improvement.

- The cost data in the beginning of the study period were unstable; to account for this, we performed several sensitivity analyses using variations in the cost estimation

than tripled to around 16000 , and its incidence more than doubled. The incidence is expected to increase further which, in combination with a growing and ageing population, will probably lead to a higher demand for THAs. As a result, the burden on healthcare resources and hospital budgets will also increase. ${ }^{1}$ According to our calculations, the mean cost for a THA in 2012 was around SEK 83000 (approximately $€ 8800$ using the average exchange rate between 2005 and 2012; $€ 1 \approx$ SEK 9.4). Given the increasing demand for THAs and that the procedure is rather costly combined with limited resources available for healthcare, a need to increase productivity exists. Hence, there is a pressure on orthopaedic departments to provide this procedure at minimum costs and with good outcomes while maintaining or increasing access. It is thus of high policy and management relevance to study the development of productivity in the provision of THA over time.

Basically, productivity is expressed as a ratio of output (eg, number of patients or 
operations) per unit of input (eg, costs) in the production process. Productivity change thus relates to a change in this ratio from one period to the next. A breakdown of the productivity change into components of change in efficiency and technology is useful, especially for policy purposes, as it provides more detailed information which can help improve productivity. Here, efficiency change relates to changes in the observed production relative to best possible production. This change is also expressed as catch-up effects where less efficient units improve their efficiency in relation to the most efficient units. Technical change describes a change in the amount of output produced from the same amount of input (or vice versa) for the most efficient departments, and may be due to, for instance, innovation.

There is an extensive body of literature on the measurement of productivity in healthcare, but few of these studies are about the development of productivity, disease based, and within the Swedish context. In 2008, Hollingsworth ${ }^{2}$ systematically reviewed over 300 published papers in the area, covering various techniques and applications. In comparison to a previous systematic review from 1999 by Hollingsworth $e t a l,{ }^{3}$ it is clear that the number of studies on the development of productivity in healthcare has increased. The number of studies is still, however, relatively low. Moreover, productivity can be analysed at the system, subsector or disease level. By focusing on a specific disease or surgical procedure, it is possible to compare homogeneous units (eg, orthopaedic departments providing THA), which reduces the risk of confounding production technologies. ${ }^{4}$ Furthermore, the managers in charge of the departments under analysis could benefit from more relevant and useful information. Nevertheless, relatively few of the previous studies were disease based, ${ }^{2}$ and previous studies in Sweden are furthermore outdated. $^{4-9}$ To the best of our knowledge, there are no existing studies to date on the development of productivity in the provision of THA.

The aim of this study was to analyse the productivity development, including changes in efficiency and technology, between 2005 and 2012 in the provision of THA in Sweden. Given the increasing demand and relatively high costs for THA, it is important to improve its productivity. Through this analysis, insight into the development of productivity, efficiency and technology in the provision of THA is provided, which can assist managers and policymakers in undertaking measures for improvement.

\section{METHODS}

\section{Database}

We used the Swedish Hip Arthroplasty Register (SHAR) to identify and collect data on patients who underwent elective primary THA due to osteoarthritis between 2005 and 2012. SHAR started in 1979 and is one of the oldest national quality registers. The register has been collecting patient-level data (eg, age, sex, diagnosis and type of implant) from all public and private orthopaedic departments in Sweden performing THA for over 25 years (ie, the degree of coverage is 100\%). According to annual validations, the completeness is $97.0 \%-98.5 \%$. Additionally, administrative data on these patients' hospital inpatient stays related to the operation were collected from the National Patient Register (NPR) provided by the National Board of Health and Welfare. These data, including information on dates of admission and discharge, readmissions, diagnoses, procedures and diagnosis-related groups (DRGs), were linked to SHAR through personal identification numbers. The data were thereafter anonymised. The study and pooling of the data were approved by the Regional Ethical Review Board in Gothenburg, Sweden.

\section{Selection of departments}

All orthopaedic departments performing THA in Sweden from 2005 to $2012(\mathrm{~N}=86)$ were eligible for inclusion in the study. However, as data from both SHAR and NPR were needed, one prerequisite for the inclusion of a department was the capability to match the data between the registers. Departments with no or low completeness in NPR (due to some under-reporting from private departments) were therefore excluded from the study. Furthermore, departments which opened during the last year or closed during the first year of the study period were excluded as data for at least 2 years were needed to be able to estimate any productivity changes. One department was further excluded as data were missing in NPR for half of the study period. Moreover, there were a few reorganisations involving changed ownership or managerial structure, mergers and splits over the time period. For simplicity, departments which merged or split over time were handled as one unit during the full study period. That is, if two departments merged after the beginning of the study period, we considered them to be one (the same) unit from the start. Similarly, if two departments split after the beginning of the study period, we considered them to be one (the same) unit after the split. Departments with changed ownership or managerial structure were also considered to be the same unit over time in the analysis. After these exclusions and reorganisations, a total of 65 orthopaedic departments remained in the study (figure 1).

\section{Input and output variables}

To facilitate measurement of productivity development of THA, we initially specified inputs and outputs in the production process of the orthopaedic departments. Three different outputs based on the types of operations used for primary THAs in Sweden were defined; the number of non-cemented, hybrid and cemented THAs per department and year fulfilling the specific inclusion and exclusion criteria listed in Box 1. Patients who underwent bilateral THA or had a prior THA within 90 days of the operation were excluded due to a statistical merging problem. Moreover, patients with a missing or incorrect 


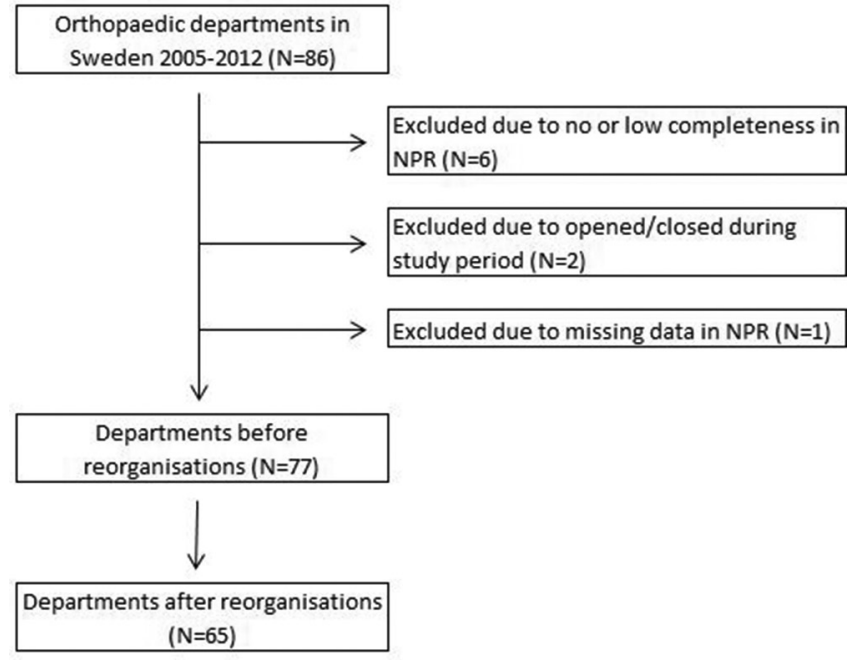

Figure 1 Department flow diagram. NPR, National Patient Register.

DRG code were excluded as we could not estimate their costs without a DRG code.

As a single input, the estimated department cost of THA was used. Following Iversen et $a l,{ }^{10}$ we considered a fixed cost for the operation and a variable cost corresponding to the length of the hospital stay (LOS) in conjunction with the operation. These costs, presented in Supplementary Table 1, were based on national averages for the DRGs including THA and were provided by the National Board of Health and Welfare. It was not possible to differentiate the fixed cost between the three different types of operations (non-cemented, hybrid and cemented THAs). As a consequence, we had to use the same fixed cost for all three types of operations. The variable cost was derived by multiplying the cost per day with the LOS for each operation. According to Supplementary Table 1, the cost data seemed to be somewhat unstable during the first years of the study period. Both cost items decreased drastically from 2005 to 2006 and were followed by a considerable increase from 2006 to 2007. To address this issue, variations in the cost calculation were performed as sensitivity analyses.

In addition to the estimated cost of the surgical episode, costs of adverse events (AEs) within 90 days $^{11}$ following

\section{Box 1 Inclusion and exclusion criteria: THA.}

Inclusion criteria

- Patient underwent a primary THA

- Patient had osteoarthritis as main diagnosis (ICD-10 code: M16*)

- Patient was 18 years of age or above

Exclusion criteria

- Patient underwent a bilateral THA

- Patient underwent a prior THA within 90 days of THA

- DRG code is missing or incorrect

DRG, diagnosis-related groups; ICD-10, International Statistical Classification of Diseases and Related Health Problems - 10th Revision; THA, total hip arthroplasty.
THA were added, if there were any. By the concept, AEs implicate all forms of rehospitalisation that might have depended on the intervention that was carried out and, in that case, not only local complications but also general medical complications and death. Following SHAR's definition of AEs within 90 days after THA, ${ }^{11}$ we included all forms of further surgery of the hip as well as cardiovascular, cerebrovascular and thromboembolic complications, pneumonia and ulcers if these complications resulted in hospitalisation as AEs. Costs for AEs were calculated using the DRG system. For each hospital stay with an $\mathrm{AE}$, the corresponding DRG weight, provided by the National Board of Health and Welfare, was multiplied with the yearly specific estimated cost of DRG weight $=1$. To avoid double counting of costs, we excluded AEs which occurred during the same hospital stay as the operation. All costs were summarised per department and year and adjusted to 2012's prices (in SEK) using the county council price index. ${ }^{12}$

\section{Data analysis}

The productivity development was measured by Malmquist Productivity Index, which was estimated following the approach developed by Färe $e t a l .^{9}$ The methodology combines ideas from Farrell's work on the measurement of efficiency, ${ }^{13}$ productivity measurement as expressed by Caves $e t a l^{14}$ and the use of distance functions as proposed by Malmquist. ${ }^{15}$ The index was calculated for each department by relating changes in their production from one period to the next, as well as by comparing their production to the best practice, that is, the departments which produces the most outputs from a given amount of input. The production of these departments creates the so-called production frontier, which accordingly shows the maximum possible production level of outputs from a specific amount of input. Due to innovation, for instance, the frontier shifts over time. Thus, the productivity development, measured by the Malmquist Productivity Index, can be split into two components: efficiency change (which relates to changes in the observed production relative to the maximum potential production, ie, the catch-up effect) and technical change (which relates to shifts in the production frontier, eg, because of innovation). As our input consisted of costs for THA, we took the approach of a cost-based index, which has previously been used by Färe et al. ${ }^{7}$ We assumed the managers have more control over the costs in the production process and might be able to reduce them while the number of operations is rather uncontrollable due to patient demand and referrals from doctors. Therefore, an input-oriented index was employed in the study. The mathematical expression of the index (Equation 1) was defined by Färe et $a^{\theta}$ as

$$
\begin{aligned}
& M\left(y^{t+1}, \quad x^{t+1}, \quad y^{t}, \quad x^{t}\right)= \\
& \frac{D^{t+1}\left(y^{t+1}, x^{t+1}\right)}{D^{t}\left(y^{t}, x^{t}\right)} \times\left[\left(\frac{D^{t}\left(y^{t+1}, x^{t+1}\right)}{D^{t+1}\left(y^{t+1}, x^{t+1}\right)}\right)\left(\frac{D^{t}\left(y^{t}, x^{t}\right)}{D^{t+1}\left(y^{t}, x^{t}\right)}\right)\right]^{1 / 2}(1)
\end{aligned}
$$


where the index $M$ measures the productivity change between the two periods $t$ and $t+1, D$ represents the Shephard ${ }^{16}$ distance functions used to calculate the productivity change from period $t$ to $t+1, x^{t}$ denotes the input vector in period $t, x^{t+1}$ denotes the input vector in period $t+1, y^{t}$ denotes the output vector in period $t$ and $y^{t+1}$ denotes the output vector in period $t+1$. The distance functions were estimated using data envelopment analysis, ${ }^{13} 17$ and a constant returns to scale technology was further assumed, which means that increases in inputs and outputs are proportionate. The first ratio in the equation above represents the change in efficiency from period $t$ to period $t+1$, whereas the ratio in the brackets measures the technical change between period $t$ and $t+1$. To conform to standard interpretation in the productivity literature, we took the inverse of the index and its components. Thus, a value $=1$ indicates no change in production, a value $<1$ indicates regress and a value $>1$ indicates progress. ${ }^{9}$

To test the null hypothesis of no change in productivity, efficiency and technology between two consecutive years, we used a bootstrap methodology developed by Simar and Wilson ${ }^{1819}$ to provide $95 \%$ CIs around the index and its components. If the CI includes unity (ie, $0 \%$ change in productivity, eg), the null hypothesis cannot be rejected and we say that the results are statistically insignificant. The calculations of the productivity development were performed using the FEAR package in the R software. ${ }^{20}$

\section{Sensitivity analyses}

To assess the robustness of the results, six different sensitivity analyses were performed (Supplementary Table 2). Because the productivity assessment is rather sensitive to the number of inputs and outputs, the three outputs were consolidated into one output (ie, the total number of THAs) in the first sensitivity analysis (S1). In the second analysis, costs for AEs were excluded (S2). To address the issue of unstable THA costs, two variations of the cost calculation were conducted in a third and fourth sensitivity analyses (S3 and S4). In S3, we used the department mean cost of THA to construct a model which we used to predict the cost of THA. These costs were collected from the cost per patient database provided by the Swedish Association of Local Authorities and Regions. ${ }^{21}$ The model included information on type of operation (non-cemented, hybrid and cemented THA), age group and LOS in conjunction with the operation. Costs for AEs were calculated using the DRG system in the same way as for the main model. In S4, following Iversen et al, ${ }^{10}$ costs for both the operation and AEs were estimated using the DRG system. For each DRG in the sample, we calculated the mean number of DRG points per day. Thereafter, we multiplied this mean with the LOS for each hospital stay to obtain the number of DRG points per stay. This number was then multiplied with the yearly specific value for DRG weight $=1$, which gave us the estimated cost for every individual hospital stay in the sample. In the fifth sensitivity analysis, we considered the LOS in conjunction with the operation, as well as the LOS with an AE diagnosis within 90 days following THA as the input (S5). Outliers were removed from the last sensitivity analysis (S6). Outliers were detected using the super-efficiency procedure with a screen level of $1.2 .{ }^{22}$ We identified 1-2 outliers per year.

\section{Patient and public involvement}

Patients and the public were not involved in the design and performance of the study. The study is based on administrative and public data and since the results are presented at department level, the patients included in the registers should not be caused by any type of discomfort or integrity infringement.

\section{RESULTS}

Descriptive statistics of the inputs and outputs are presented in table 1 . The average number of THAs, specifically non-cemented and hybrid THAs, increased over the time period. In 2005, the average number of THAs for a department was 157 , and in 2012, the corresponding figure was 187. According to the input variables, there were no clear trends of costs over time. The total cost for the average department, including costs for all THAs and AEs, was the highest in 2009 (around SEK 17 million) and the lowest in 2006 (SEK 12 million). Similarly, the average total cost for all THAs only (AEs excluded) was highest in 2009 and lowest in 2006. The mean cost of a single THA, however, seems to have decreased over time, from SEK 93 000 in 2005 to SEK 83000 in 2012. This trend explains why an increase in the total costs for THAs did not occur, even though the number of THAs increased. The average total cost for all AEs, as well as the mean number of AEs, was rather stable over time, around SEK 500 000, respectively, $7-9$ AEs (around $4 \%-5 \%$ of the operations) per year.

The results are presented in table 2, where averages of changes in productivity (Malmquist Productivity Index), efficiency and technology, calculated as the geometric mean of the results from the 65 departments, are displayed. Recall that a value $=1$ indicates no change in production, a value $<1$ indicates regress and a value $>1$ indicates progress between two consecutive years.

The results indicate significant improvement in productivity in three periods, by $27 \%$ (CI: 26.0 to 27.0 ) from 2005 to $2006,11.0 \%$ (CI: 10.3 to 11.2 ) from 2009 to 2010 and $1.6 \%$ (CI: 1.2 to 1.9 ) from 2010 to 2011 . A significant productivity regress was found in four periods: $12.1 \%$ (CI: 12.1 to 13.2 ) from 2006 to $2007,10.5 \%$ (CI: 10.3 to 11.0 ) from 2007 to $2008,0.8 \%$ (CI: 0.7 to 1.5 ) from 2008 to 2009 and $1.7 \%$ (CI: 1.5 to 2.1) from 2011 to 2012. For the time span as a whole, we noted an average productivity increase of $1.4 \%$ per year, where changes in efficiency (ie, the catching-up effects of departments), contributed more to the improvement $(1.1 \%)$ than did technical change $(0.2 \%)$. The increase in productivity was expected given that the mean cost of a THA decreased 
Table 1 Descriptive statistics, inputs and outputs; department mean (arithmetic), minimum and maximum values, 65 departments each year, 2005-2012

\begin{tabular}{|c|c|c|c|c|c|c|c|c|c|c|}
\hline & & & 2005 & 2006 & 2007 & 2008 & 2009 & 2010 & 2011 & 2012 \\
\hline \multirow[t]{15}{*}{ Input ${ }^{\star}$} & \multirow{3}{*}{ Total cost (all THAs+AEs) } & Min. & 843 & 978 & 713 & 687 & 1987 & 2171 & 1300 & 1873 \\
\hline & & Mean & 14923 & 12000 & 13628 & 15471 & 17080 & 15603 & 15251 & 15593 \\
\hline & & Max. & 56869 & 48858 & 65277 & 68573 & 67218 & 55769 & 52913 & 41779 \\
\hline & \multirow[t]{3}{*}{ Total cost (all THAs) } & Min. & 843 & 796 & 713 & 687 & 1987 & 2024 & 1300 & 1842 \\
\hline & & Mean & 14446 & 11477 & 13132 & 14895 & 16500 & 15076 & 14726 & 15074 \\
\hline & & Max. & 54622 & 46562 & 62135 & 65988 & 64509 & 53799 & 51103 & 40608 \\
\hline & \multirow[t]{3}{*}{ Total cost (all AEs) } & Min. & 0 & 0 & 0 & 0 & 0 & 35 & 0 & 0 \\
\hline & & Mean & 477 & 523 & 497 & 576 & 581 & 527 & 525 & 519 \\
\hline & & Max. & 2515 & 2297 & 3141 & 2584 & 2709 & 1970 & 1811 & 1868 \\
\hline & \multirow[t]{3}{*}{ Number of AEs } & Min. & 0 & 0 & 0 & 0 & 0 & 1 & 0 & 0 \\
\hline & & Mean & 7 & 8 & 8 & 9 & 9 & 9 & 8 & 8 \\
\hline & & Max. & 36 & 39 & 46 & 43 & 41 & 37 & 28 & 31 \\
\hline & \multirow[t]{3}{*}{ Mean cost of a THA } & Min. & 70 & 54 & 61 & 67 & 70 & 66 & 63 & 65 \\
\hline & & Mean & 93 & 72 & 82 & 91 & 92 & 82 & 81 & 83 \\
\hline & & Max. & 120 & 90 & 104 & 121 & 132 & 115 & 109 & 113 \\
\hline \multirow[t]{12}{*}{ Output } & \multirow[t]{3}{*}{ All types of THAs } & Min. & 11 & 13 & 8 & 8 & 19 & 19 & 13 & 18 \\
\hline & & Mean & 157 & 162 & 162 & 167 & 184 & 187 & 186 & 187 \\
\hline & & Max. & 620 & 681 & 776 & 769 & 774 & 716 & 700 & 588 \\
\hline & \multirow[t]{3}{*}{ Cemented THAs } & Min. & 6 & 3 & 3 & 6 & 11 & 11 & 8 & 8 \\
\hline & & Mean & 133 & 134 & 126 & 124 & 135 & 134 & 129 & 129 \\
\hline & & Max. & 613 & 657 & 707 & 669 & 672 & 564 & 526 & 398 \\
\hline & \multirow[t]{3}{*}{ Non-cemented THAs } & Min. & 0 & 0 & 0 & 0 & 0 & 0 & 0 & 0 \\
\hline & & Mean & 12 & 16 & 19 & 23 & 26 & 27 & 29 & 29 \\
\hline & & Max. & 90 & 119 & 123 & 107 & 109 & 125 & 133 & 162 \\
\hline & \multirow[t]{3}{*}{ Hybrid THAs } & Min. & 0 & 0 & 0 & 0 & 0 & 0 & 0 & 0 \\
\hline & & Mean & 12 & 12 & 16 & 20 & 24 & 26 & 27 & 29 \\
\hline & & Max. & 106 & 107 & 172 & 224 & 203 & 210 & 218 & 260 \\
\hline
\end{tabular}

${ }^{*}$ In SEK 1000 (2012's prices).

AEs, adverse events; THA, total hip arthroplasty.

over the study period at the same time as costs for AEs were stable (table 1$)$.

Furthermore, the results of the breakdown of the index show a significant positive technical change in three periods (16.9\%; CI: 9.8 to 18.7 between 2005 and 2006, 3.2\%; CI: 1.7 to 7.8 between 2008 and 2009 and $9.1 \%$; CI: 5.1 to 11.2 between 2009 and 2010) and a significant negative technical change in two periods (12.6\%; CI: 10.5 to 15.2 between 2006 and 2007 and 10.2\%; CI: 7.7 to 12.5 from 2007 to 2008). Significant progress in efficiency was achieved in two periods (8.7\%; CI: 6.9 to 16.3 from 2005 to 2006 and 2.6\%; CI: 1.0 to 5.9 from 2010 to 2011), whereas a significant regress was achieved in one period (3.9\%; CI: 2.7 to 7.9 from 2008 to 2009 ).

Inspection of the department-specific results (Supplementary Tables 3-5) showed that none of the departments progressed or regressed in productivity, efficiency or technology in all seven periods. There was only one department (no. 43) with statistically significant improvement in its productivity in six periods. Four departments were efficient in five or six periods (nos. 8, 9, 26 and 39). Almost all departments improved their productivity between 2005 and 2006, as well as between 2009 and 2010 . There was also a positive change in technology for almost all departments during the same time periods.

\section{Sensitivity analyses}

According to the sensitivity analysis, the results seem to be rather robust to outlier removal, different model specifications and variations in the cost calculations (Supplementary Table 6 ). In all models, the overall productivity slightly increased over the time period. Changes in efficiency contributed more to this progress than did technical change in all models but S1, S5 and S6, where the components almost equally contributed to the progress. In S5, the results deviated somewhat from those of the 
Table 2 Malmquist Productivity Index, efficiency change and technical change; mean (geometric), 2005-2012

\begin{tabular}{llll}
\hline Period & Malmquist Productivity Index & Efficiency change & Technical change \\
\hline $2005-2006$ & $1.270(27.0)^{\star}$ & $1.087(8.7)^{\star}$ & $1.169(16.9)^{\star}$ \\
$2006-2007$ & $0.879(-12.1)^{\star}$ & $1.006(0.6)$ & $0.874(-12.6)^{\star}$ \\
$2007-2008$ & $0.895(-10.5)^{\star}$ & $0.996(-0.4)$ & $0.898(-10.2)^{\star}$ \\
\hline $2008-2009$ & $0.992(-0.8)^{\star}$ & $0.961(-3.9)^{\star}$ & $1.032(3.2)^{\star}$ \\
$2009-2010$ & $1.110(11.0)^{\star}$ & $1.018(1.8)$ & $1.091(9.1)^{\star}$ \\
$2010-2011$ & $1.016(1.6)^{\star}$ & $1.026(2.6)^{\star}$ & $0.990(-1.0)$ \\
$2011-2012$ & $0.983(-1.7)^{\star}$ & $0.988(-1.2)$ & $0.995(-0.5)$ \\
\hline Mean & $1.014(1.4)$ & $1.011(1.1)$ & $1.002(0.2)$ \\
\hline
\end{tabular}

Malmquist Productivity Index is defined as the mathematical product of efficiency change and technical change. Due to rounding, there are minor deviations from this identity in the table. As is common in bootstrapped Malmquist analyses, we found that the bias-corrected estimates had a larger estimated mean squared error than the original deterministic estimates, and following Simar and Wilson, ${ }^{18}$ the uncorrected estimates are reported in the table. A value $=1$ indicates no change between two consecutive years, $>1$ indicates progress and $<1$ indicates regress. Numbers in parentheses indicate percentage change.

${ }^{*}$ indicates statistically significantly different from 1.0 at the $95 \%$ level.

other analyses, with an average productivity increase in $4.2 \%$ per year compared with an annual average increase in $1.4 \%$ to $1.8 \%$. In S5, LOS constituted the input rather than costs. This result is, however, not surprising as LOS decreased in a larger magnitude than THA costs over the time period.

\section{DISCUSSION}

This register-based study aimed to analyse the development of productivity, as well as changes in efficiency and technology, between 2005 and 2012 for orthopaedic departments performing THA in Sweden. A slight improvement of productivity over time was found, which was mainly due to progress in efficiency rather than positive technical changes. A contribution to this catch-up effect could be the sharing of information and the annual tradition of public benchmarking of procedures, volumes and quality indicators between all Swedish orthopaedic departments, which SHAR has provided for a long time with the aim to improve the performance of THA. Thus, a possible explanation of this progress is the transparency of performance, by which the departments could learn from each other, and most likely, the less efficient departments are put under pressure to improve.

As previously mentioned, technical change relates to shifts in the production frontier (eg, because of innovation). Swedish orthopaedic providers have been rather conservative in terms of adapting new prostheses for THA, so the positive shift of the frontier in this study might instead be explained by the decreased cost of THA over time. Given that the cost of THA (our input) is partly based on the LOS in conjunction with the operation, which has decreased over time, ${ }^{11}$ the positive technical change found here might refer to shorter LOSs over time. However, this development has been counteracted by an increase in the cost per bed-day as the service per day has been more intensive.
To our knowledge, this is the first study to use the Malmquist Productivity Index to study productivity development in THA. The overall results showing productivity improvements are, however, in line with previous studies in Sweden on productivity development in other specialties, although the contributions to the improvement differ. For example, Tambour $^{4}$ found several annual positive productivity changes in the ophthalmology sector between 1988 and 1993, although these improvements were mainly due to positive changes in technology. The introduction of new administrative systems and improvements in medical technology were discussed as possible factors explaining the positive shifts in the frontier technology. However, while controlling for the former factor to some extent, no evidence was found that this factor explained the shifts. Improvements in medical technology were difficult to control for in the analysis, and the effect was thus not possible to evaluate. Similarly, Färe et $a l^{9}{ }^{9}$ who assessed productivity changes in pharmacies in Sweden between 1980 and 1989, found increased productivity in seven time periods, where progress in technology also mainly contributed to the productivity gains at the end of the 1980s. Furthermore, in our study, none of the departments that were efficient in most of the periods were continuously progressing in technology during the corresponding periods. This implies that none of the included departments could be identified as a 'technical' leader, similar to what Linna ${ }^{23}$ found when studying overall hospital efficiency in Finland.

In 2009, there was a policy reform introduced for hip and knee arthroplasty in the largest region in Sweden. Through the reform, a bundle payment model, patient choice and free establishment of new providers were introduced to the market with the aim to improve performance through competition. The present study found a large increase in productivity between 2009 and 2010, and it would therefore be interesting in a future study to analyse whether this increase was an effect of the reform. 
In the present study, the development of productivity, efficiency and technology in the provision of THA in Sweden was analysed based on patient-level data collected from SHAR and NPR. The study included almost all orthopaedic departments in Sweden, and the results give a picture of the overall development over time. The generalisability of the results to other countries depends on, inter alia, the specific country's healthcare system, financing, reimbursement, the history of working with registers and national benchmarking. Given the similarities in these aspects between the Scandinavian countries, the results might be generalisable to these countries.

A limitation of the study is the unstable cost data for THA in the beginning of the study period. Therefore, the drastic changes in this period should be interpreted with caution. Nevertheless, the sensitivity analyses showed that the overall results were rather robust to variations in the cost calculations.

Differences in productivity and its components both between departments and within departments over time were further revealed. To be able to improve the overall productivity, it is important to address these differences and reduce the variation by improving the performance of the least productive departments. Two possible sources of differences in productivity across the departments are variation in quality of care and variation in patient case mix. Given that the main purpose of this study was limited to analysing the overall development of productivity over time, differences in performance across departments were not analysed, and differences in quality and case mix were not considered. However, a future study which measures and controls for quality of care, including patient-reported outcomes, and patient case mix with the aim to address and explain the differences would thus be important and interesting.

\section{CONCLUSIONS}

Our study provided insight into the development of productivity in the provision of THA, which can assist managers and policymakers in undertaking measures for improvement. We found a slight improvement of productivity over time, which was mainly driven by changes in efficiency. Further research is, however, needed where differences in quality of care and patient case mix between departments are taken into account.

Acknowledgements We acknowledge Region Stockholm for funding support. We are grateful to SHAR for allowing us to use their data. Moreover, we are thankful for the valuable comments from the reviewers, as well as for comments on an earlier version of the manuscript received at a seminar at Karolinska Institutet.

Contributors FG came up with the original idea, designed the study, performed data management and data analysis, interpreted the results and drafted the manuscript. GG supported data collection and interpreted the results. SK supported data analysis and interpreted the results. SN supported data collection. CR came up with the original idea, supported data collection and interpreted the results. All authors reviewed the manuscript.
Funding This study was supported by unrestricted grants provided by Region Stockholm.

Competing interests None declared.

Patient consent for publication Not required.

Provenance and peer review Not commissioned; externally peer reviewed.

Data availability statement Data may be obtained from a third party and are not publicly available.

Open access This is an open access article distributed in accordance with the Creative Commons Attribution Non Commercial (CC BY-NC 4.0) license, which permits others to distribute, remix, adapt, build upon this work non-commercially, and license their derivative works on different terms, provided the original work is properly cited, appropriate credit is given, any changes made indicated, and the use is non-commercial. See: http://creativecommons.org/licenses/by-nc/4.0/.

\section{REFERENCES}

1. Nemes S, Gordon M, Rogmark C, et al. Projections of total hip replacement in Sweden from 2013 to 2030. Acta Orthop 2014;85:238-43.

2. Hollingsworth $B$. The measurement of efficiency and productivity of health care delivery. Health Econ 2008;17:1107-28.

3. Hollingsworth B, Dawson PJ, Maniadakis N. Efficiency measurement of health care: a review of non-parametric methods and applications. Health Care Manag Sci 1999;2:161-72.

4. Tambour M. The impact of health care policy initiatives on productivity. Health Econ 1997;6:57-70.

5. Roos P. Measurement of productivity in hospital services using Malmquist index approaches: A discussion of methods and illustration to eye surgery.. In: CSLS conference on service sector productivity and the productivity paradox. Canada, 1997.

6. Lothgren M, Tambour M. Bootstrapping the data envelopment analysis Malmquist productivity index. Appl Econ 1999;31:417-25.

7. Färe R, Grosskopf S, Roos P. Produktivitetsutvecklingen inom sjukhussektorn 1970-1992 [Productivity growth in the hospital sector 1970-1992]. Finansdepartementet (Ministry of Finance). Ds Fi $1994 ; 71$.

8. Färe R, Grosskopf S, Lindgren B, et al. Productivity developments in Swedish hospitals: a Malmquist output index approach. in: data envelopment analysis: theory, methodology, and applications. Springer 1994:253-72.

9. Färe R, Grosskopf S, Lindgren B, et al. Productivity changes in Swedish pharamacies 1980?1989: A non-parametric Malmquist approach. J Prod Anal 1992;3:85-101.

10. Iversen $\mathrm{T}$, Aas E, Rosenqvist $\mathrm{G}$, et al. Comparative analysis of treatment costs in EUROHOPE. Health Econ 2015;24(Suppl 2):5-22.

11. Swedish Hip Arthroplasty Register. Annual report 2013, 2014.

12. Information Om prisindex LPI och LPIK, 2018. Available: https://skl. se/ekonomijuridikstatistik/ekonomi/budgetochplanering/prisindex/ Ipiochlpik.1332.html

13. Farrell MJ. The measurement of productive efficiency. $J R$ Stat Soc Ser A 1957;120:253-90.

14. Caves DW, Christensen LR, Diewert WE. The economic theory of index numbers and the measurement of input, output, and productivity. Econometrica 1982;50:1393-414.

15. Malmquist S. Index numbers and indifference surfaces. Trabajos de Estadistica 1953;4:209-42.

16. Shephard RW. Theory of cost and production functions. Princeton University Press, 1970.

17. Charnes A, Cooper WW, Rhodes E. Measuring the efficiency of decision making units. Eur J Oper Res 1978;2:429-44.

18. Simar L, Wilson PW. A general methodology for bootstrapping in non-parametric frontier models. J App/ Stat 2000;27:779-802.

19. Simar L, Wilson PW. Estimating and bootstrapping Malmquist indices. Eur J Oper Res 1999;115:459-71.

20. Wilson PW. Fear: a software package for frontier efficiency analysis with R. Socioecon Plann Sci 2008;42:247-54.

21. Swedish Association of Local Authorities and Regions. KPP Databas, 2018. Available: https://skl.se/ekonomijuridikstatistik/statistik/ kostnadperpatientkpp/kppdatabas.1079.html

22. Banker RD, Chang $H$. The super-efficiency procedure for outlier identification, not for ranking efficient units. Eur $J$ Oper Res 2006;175:1311-20.

23. Linna M. Measuring hospital cost efficiency with panel data models. Health Econ 1998;7:415-27. 